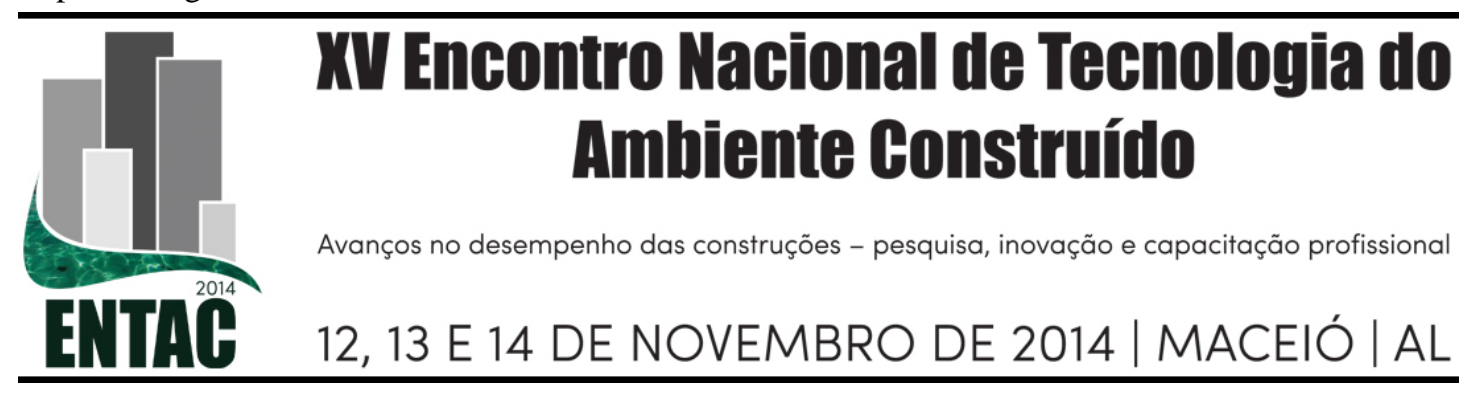

\title{
ESTUDO DO EFEITO DE PONTES TÉRMICAS EM ESTRUTURAS DE CONCRETO ARMADO NO DESEMPENHO ENERGÉTICO DE EDIFÍCIO HOTELEIRO PARA 6 ZONAS BIOCLIMÁTICAS BRASILEIRAS
}

\author{
GIOIELLI, Beatriz (1); CUNHA, Eduardo Grala (2); \\ (1) Acadêmica, curso de Arquitetura e Urbanismo/ UFPEL, beagioielli@gmail.com, \\ (2) Prof. Dr. Arquiteto e Urbanista; Departamento de Tecnologia da Construção - Faculdade de \\ Arquitetura e Urbanismo/UFPel, eduardogralacunha@yahoo.com.br
}

\begin{abstract}
RESUMO
O objetivo do artigo é apresentar a análise da influência das pontes térmicas de sistemas estruturais em concreto armado no desempenho energético da envoltória de um edifício comercial localizado nas zonas bioclimáticas brasileiras de 1 a 6 utilizando simulação computacional.O método utilizado para alcançar o objetivo é caracterizado pelas seguintes etapas: 1) Definição e configuração do caso base; 2) Definição e configuração do modelo de referência; 3) Otimização das simulações e 4) Simulação e comparação do consumo do caso base e do modelo de referência. Como principais resultados observou-se que as pontes térmicas de concreto armado interferem no desempenho energético da edificação e o impacto relaciona-se ao percentual de abertura transparente (PAFT) da edificação. Para edificações de hotelaria com PAFT de $30 \%$ e $45 \%$ as pontes térmicas implicam em uma diminuição da estimativa de consumo, que dependendo da zona bioclimática pode chegar a $10 \%$. No caso de $60 \%$ de PAFT a não consideração da ponte térmica pode representar um aumento de até $4 \%$ na estimativa de consumo dependendo da zona bioclimática brasileira.
\end{abstract}

Palavras-chave: Ponte térmica, desempenho energético, eficiência energética.

\begin{abstract}
This article aims to present an analysis of the influence of thermal bridges on reinforced concrete structural systems regarding energy performance of the envelope of a commercial building located in the bioclimatic zones 1 to 6 in Brazil, using computer simulation. The method used to achieve this goal includes the following steps: 1) Definition and configuration of the base case; 2) Definition and configuration of the reference model; 3) Optimization of energy modeling and 4) Energy modeling and comparison of consumption between the base case and the reference model. Main results showed that thermal bridges in reinforced concrete interfere on the building's energy performance and that the impact is related to the window-to-wall ratio (WWR) on the building. For hotel buildings with WWR from $30 \%$ to $45 \%$, thermal bridges imply a decrease in estimated consumption, which can reach $10 \%$, depending on the bioclimatic zone. For $60 \%$ WWR, the non-consideration of thermal bridges can represent up to $4 \%$ of increase in estimated consumption, depending on the Brazilian bioclimatic zone.
\end{abstract}

Keywords: Thermal bridge, thermal performance, energy efficiency.

\section{INTRODUÇÃO}

No Brasil as novas normas de desempenho e eficiência energética são recentes, e ainda não impactam a vida dos brasileiros de forma incisiva. Convivemos ainda, em alguns 
casos, com técnicas e sistemas construtivos inadequados para os diferentes contextos climáticos encontrados no país, gerando muitas vezes a ocorrência de desconforto térmico para os usuários. O aumento do poder de compra do brasileiro nos últimos anos aliado a soluções construtivas inadequadas, do ponto de vista bioclimático, mudaram a maneira que as edificações consomem energia. Nos últimos anos foram publicadas as primeiras normas de desempenho térmico e eficiência energética de edificações. A Associação Brasileira de Normas Técnicas (ABNT) aprovou duas normas: a NBR 15220 - Desempenho Térmico de Edificações - em 2005 e a NBR 15575 - Desempenho de Edifícios Habitacionais - em 2013.

A NBR 15575 (2013) é ampla e propõe uma análise de requisitos, critérios e métodos de avaliação de desempenho que vão desde o desempenho estrutural, estanqueidade de aberturas, desempenho térmico, acústico e lumínico, até a adequação ambiental, durabilidade e manutenibilidade da edificação. A NBR 15220 (2005), em sua parte 3, define recomendações quanto ao desempenho térmico de habitações unifamiliares de interesse social, indicando as diretrizes construtivas e as estratégias bioclimáticas mais adequadas para diversas Zonas Bioclimáticas. Contudo, muitas das prescrições contidas na norma, principalmente em relação às características físicas dos fechamentos (transmitância térmica, atraso térmico e fator de calor solar), tem sido contestadas em trabalhos de pesquisadores da área.

Alguns autores como MATOS (2007) e PEREIRA (2009) provaram através de estudos que alguns dos critérios utilizados pelas normas são inadequados ou equivocados, de forma que a própria NBR 15575 (2013), posterior à NBR 15220 (2005), já utiliza alguns parâmetros diferentes para avaliação simplificada das edificações.

Foi possível constatar a importância de se ter recomendações projetuais confiáveis graças à pesquisa realizada por VENÂNCIO (2011) que comprovou que mais de 50\% dos arquitetos, consultores e pesquisadores adotam essas recomendações como a ferramenta mais frequente na tomada de decisões.

Existem vários trabalhos com o foco na análise do desempenho energético da envoltória, no entanto se concentram, principalmente, nas questões de controle de radiação solar no contexto dos fechamentos opacos e transparentes. Como a tese de doutorado de CARLO (2008) que se tornou referência porque em sua pesquisa ela realizou mais de 1000 simulações computacionais com o software Energy Plus e com base no seu trabalho foram propostas equações de regressão linear presentes no Regulamento Brasileiro de Eficiência Energética para Edifícios Comerciais, de Serviços e Públicos (RTQ-C). As equações geradas avaliam o nível de eficiência energética da envoltória de edifícios comerciais, de serviços e públicos pelo método prescritivo. No entanto, para edificações residenciais e comerciais não são encontrados trabalhos acadêmicos no Brasil cujo foco seja a análise do impacto das pontes térmicas no contexto do desempenho termo energético das edificações. Ressalta-se que a maior interferência das pontes térmicas no desempenho termoenergético de edificações ocorrem em climas frios, e no caso do contexto Brasileiro, caracterizados nas zonas bioclimáticas 1,2 e 3 .

Contudo, no âmbito internacional, observando países com rigor climático referente aos períodos de estação fria, as consequências negativas das pontes térmicas são reconhecidas e estratégias para a sua correção são utilizadas. As pontes térmicas agravam o risco da ocorrência de condensações superficiais e a formação de fungos filamentosos, e ainda por sua natureza servem como caminho para a transmissão de calor agravando as perdas térmicas para o exterior, o que poderá suscitar em um maior 
consumo energético para aquecimento. Segundo EVANS e SCHILLER (2010) as pontes térmicas favorecem a ocorrência da condensação superficial, e a norma argentina IRAM 11.549 (2002) define que a ponte térmica ocasiona maior fluxo de calor originando condensação superficial. No mesmo contexto HAUPT (2007) afirma que as pontes térmicas geram uma diminuição brusca da temperatura de superfícies podendo gerar condensação superficial. Como consequência direta da condensação superficial na maioria das vezes há formação de fungos filamentosos.

Nos estudos sobre transmissão de calor em edificações o efeito das pontes térmicas ainda é um assunto negligenciado ou não implementado corretamente nos cálculos. Isso ocorre principalmente porque a transferência de calor nas edificações vem sendo considerada como unidimensional. Segundo HAUPT (2007) a ponte térmica é caracterizada quando o fluxo térmico não é mais unidimensional. VALÉRIO (2007) afirma que a ponte térmica é toda a parte da envolvente de um edifício onde não é possível admitir a hipótese de unidirecionalidade do fluxo de calor e, por conseguinte, deixa de ser válida a abordagem de cálculo convencionalmente usado em análise de desempenho termo energético de edifícios, que toma a resistência térmica dos elementos uniforme na sua área. Em termos de fenômeno físico, nas zonas de pontes térmicas as linhas do fluxo de calor deixam de ser retilíneas, aspecto característico de um processo unidirecional, e tomam as direções em que a resistência oferecida à passagem de calor é menor (Figura 1). O software DesignBuilder permite a criação de sub-superfícies sobrepostas possibilitando a variação da resistência térmica ao longo do fechamento vertical.

Com esse raciocínio percebe-se a importância de quantificar a perda ou o ganho de calor gerado pelas pontes térmicas. Elas interferem no conforto térmico e desempenho energético de uma edificação, assim como na propensão às patologias.

Figura 1 - Exemplos de pontes térmicas no envelope construído
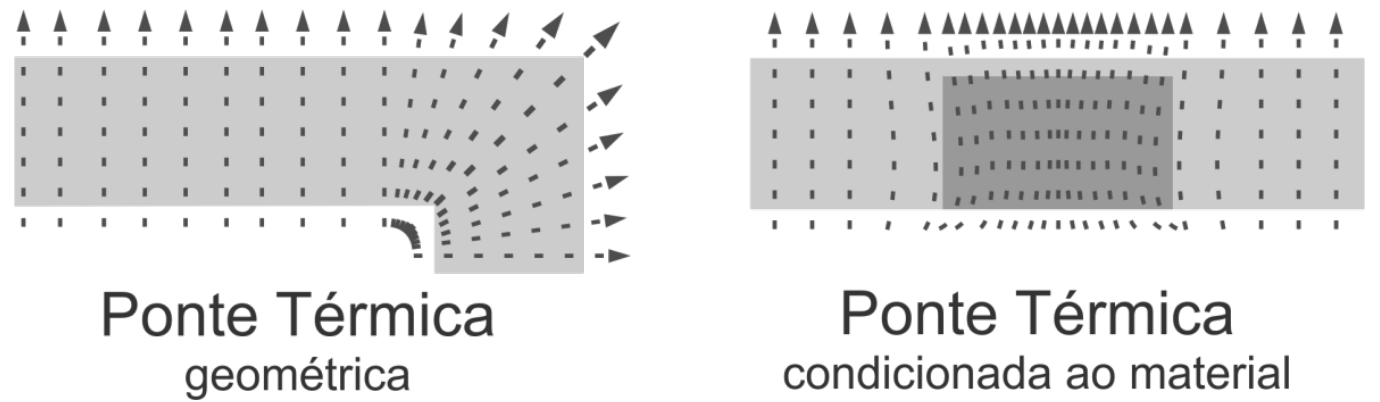

Nesse sentido o objetivo do artigo é apresentar a análise da influência das pontes térmicas de sistemas estruturais em concreto armado no desempenho energético da envoltória de um edifício hoteleiro, nas zonas bioclimáticas brasileiras de 1 a 6 utilizando simulação computacional.

\section{MÉTODO}

A pesquisa foi desenvolvida em quatro etapas. Na primeira etapa do processo se definiu a tipologia a ser analisada, observando as características de uso e ocupação como também a densidade de carga interna. A tipologia escolhida como caso de estudo foi 
uma edificação hoteleira, que conjuga a ocupação residencial com a densidade de carga interna de edifícios comerciais.

A partir da definição da tipologia, iniciou-se a segunda etapa da pesquisa: a modelagem e configuração da edificação. O programa utilizado foi o DesignBuilder versão 3.0.0.15, considerando na modelagem a presença e ausência de pontes térmicas referentes à estrutura em concreto. Devido a limitações do programa para a modelagem das pontes térmicas, foi necessária uma adaptação do modelo para que o software fosse capaz de interpretar as pontes térmicas e, para tanto, paredes equivalentes foram calculadas, caracterizado a seguir. Com o objetivo de melhorar e reduzir o tempo da realização das simulações foi desenvolvido um trabalho de otimização das simulações, descrita a seguir na terceira etapa da pesquisa.

De posse dos modelos com e sem as pontes térmicas modelados e configurados no DesignBuilder, deu-se inicio às simulações - quarta etapa da pesquisa. O modelo da edificação hoteleira foi simulado com diferentes configurações: alterações de PAFT percentual de aberturas nas fachadas $(30 \%, 45 \%, 60 \%)$, densidade de iluminação e densidade de carga de equipamentos, referenciados pela norma NBR 16401 (ABNT, 2008) e pelo RTQ-C (2010). Estes parâmetros resultaram em 36 configurações simuladas para cada contexto climático das seis Zonas Bioclimáticas Brasileiras.

\subsection{Definição e configuração do caso base}

Definido como hotel, o caso base contém características residenciais e comerciais já mencionadas anteriormente, sendo ocupadas todas as horas do dia. Com base na análise tipológica de CARLO (2008) foram definidos o volume e o percentual de área de janela na fachada. A partir dos dados obtidos gerou-se um projeto arquitetônico que posteriormente, foi modelado no programa DesignBuilder versão 3.0.0.15. O modelo foi configurado utilizando os parâmetros referentes à tipologia de hotelaria do trabalho de referência que são apresentados na Tabela 1.

\section{Quadro 1 - Parâmetros e variáveis do modelo base}

\begin{tabular}{|c|c|c|}
\hline Parâmetros & \multicolumn{2}{|c|}{ Variáveis } \\
\hline Dimensões & \multicolumn{2}{|c|}{$52 \mathrm{~m} \times 17 \mathrm{~m}$} \\
\hline Número de Pavimentos & \multicolumn{2}{|c|}{5} \\
\hline $\begin{array}{c}\text { Percentual de Área de Abertura na } \\
\text { Fachada Total }\end{array}$ & \multicolumn{2}{|c|}{$30 \%$} \\
\hline \multirow{3}{*}{ Vidro } & Cor & Incolor \\
\hline & Espessura & $4 \mathrm{~mm}$ \\
\hline & Película & Não \\
\hline \multirow{2}{*}{ Parede } & Transmitância & $1,92 \mathrm{~W} / \mathrm{m}^{2} \mathrm{~K}$ \\
\hline & Absortância & 0,25 \\
\hline Ocupação - Densidade & \multicolumn{2}{|c|}{0,28 pessoas $/ \mathrm{m}^{3}$} \\
\hline \multirow{2}{*}{ Densidade de Carga Interna } & Iluminação & $21 \mathrm{~W} / \mathrm{m}^{2}$ \\
\hline & Equipamentos & $60 \mathrm{~W} / \mathrm{m}^{2}$ \\
\hline \multirow{6}{*}{$\begin{array}{c}\text { Padrão de Uso } \\
\text { (dias úteis; sáb/dom) }\end{array}$} & 0:00-6:00 & $100 \%$ \\
\hline & 7:00-15:00 & $50 \%$ \\
\hline & 16:00-24:00 & $75 \%$ \\
\hline & 0:00-6:00 & $60 \%$ \\
\hline & 7:00-15:00 & $30 \%$ \\
\hline & 16:00-24:00 & $40 \%$ \\
\hline
\end{tabular}




\subsubsection{Definição da parede equivalente}

Para que pudessem ser configuradas as paredes externas no programa DesignBuilder, considerando a composição heterogênea do fechamento em alvenaria, foi necessária a construção de uma parede equivalente composta por: reboco, material cerâmico, camada de ar, material cerâmico e reboco. Todo o sistema foi configurado com a mesma transmitância e capacidade térmica da parede original. Na Figura 2 apresenta-se a parede equivalente.

\section{Figura 2 - Parede equivalente}

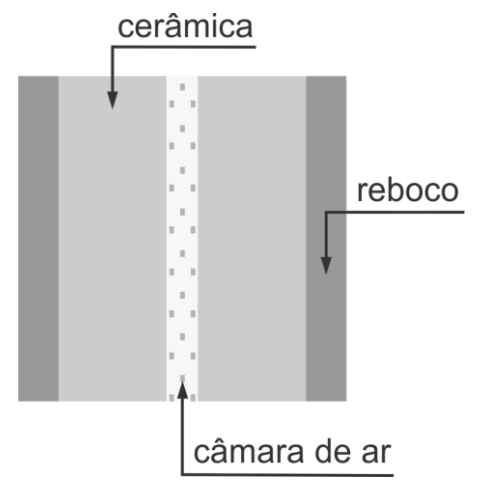

Na Tabela 2 caracterizam-se as propriedades térmicas dos materiais que compõem a parede. Considerando as alterações das espessuras, nessa nova parede equivalente, foi necessário ajustar a densidade de massa aparente do material cerâmico.

\section{Quadro 2 - Materiais e parâmetros da parede equivalente}

\begin{tabular}{|c|c|c|}
\hline \multicolumn{1}{|c|}{ Materiais } & \multicolumn{2}{|c|}{ Parâmetros } \\
\hline \multirow{2}{*}{ Sistema (Paredes) } & $\begin{array}{c}\text { Capacidade } \\
\text { Térmica }\end{array}$ & $202 \mathrm{~kJ} / \mathrm{m}^{2} \mathrm{~K}$ \\
\hline \multirow{2}{*}{ Reboco } & Espessura & $0,025 \mathrm{~m}$ \\
\cline { 2 - 3 } & $\begin{array}{c}\text { Densidade de } \\
\text { Massa }\end{array}$ & $2000 \mathrm{~kg} / \mathrm{m}^{3}$ \\
\cline { 2 - 3 } & Calor Específico & $1,00 \mathrm{~kJ} / \mathrm{kgK}$ \\
\hline \multirow{2}{*}{ Cerâmica } & Espessura & $0,076 \mathrm{~m}$ \\
\cline { 2 - 3 } & $\begin{array}{c}\text { Densidade de } \\
\text { Massa }\end{array}$ & $739,13 \mathrm{~kg} / \mathrm{m}^{3}$ \\
\cline { 2 - 3 } & Calor Específico & $0,92 \mathrm{~kJ} /(\mathrm{kgK})$ \\
\hline Câmara de Ar & Espessura & $0,02 \mathrm{~m}$ \\
\hline
\end{tabular}

\subsection{Definição e configuração do modelo de referência}

Para atingir o parâmetro de transmitância de $1,92 \mathrm{~W} / \mathrm{m}^{2} \mathrm{~K}$ determinado no caso base, e representar as pontes térmicas no programa DesignBuilder, calculou-se uma parede equivalente modelada com 3 sub superfícies (uma ferramenta de modelagem do 
programa que permite sobreposição de camadas): a parede pesada (PP), representando as vigas e pilares de concreto com reboco; parede leve (PL), composta por aglomerado e concreto, representando o fechamento em alvenaria; e a parede interna (PI), composta por concreto em toda a sua extensão, conforme mostra a Figura 3. Cabe-se ressaltar que o Energy Plus (com a interface gráfica do DesignBuilder) considera o envelope construído composto por uma ou mais camadas homogêneas. Nesse sentido para que fosse possível a criação nas paredes de transmitâncias diferenciadas para a estrutura de concreto armado e a parede de alvenaria foi necessária a configuração da parede descrita na figura 3. Na Tabela 3 estão os materiais com os seus parâmetros detalhados.

\section{Figura 3 - Parede equivalente modelada com sub-superfícies no programa}

\section{DesignBuilder}

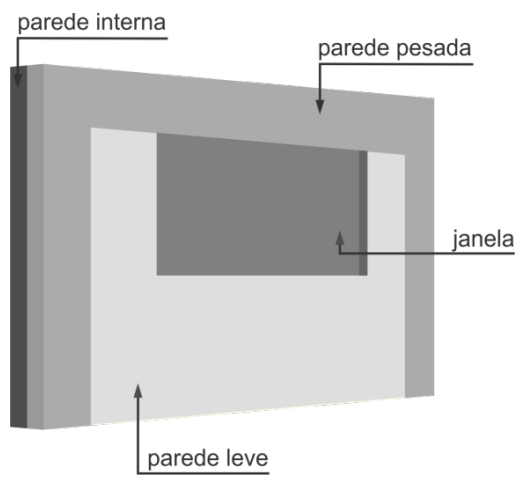

Quadro 3 - Parâmetros e variáveis do modelo base

\begin{tabular}{|c|c|c|c|}
\hline \multirow{3}{*}{ Parede Interna (PI) } & \multirow{3}{*}{ Concreto } & Condutividade Térmica & $1,75 \mathrm{~W} / \mathrm{mK}$ \\
\hline & & Espessura & $0,08 \mathrm{~m}$ \\
\hline & & Densidade & $2400 \mathrm{~kg} / \mathrm{m}^{3}$ \\
\hline \multirow{3}{*}{ Parede Leve (PL) } & \multirow{3}{*}{ Concreto } & Condutividade Térmica & $1,75 \mathrm{~W} / \mathrm{mK}$ \\
\hline & & Espessura & $0,062 \mathrm{~m}$ \\
\hline & & Densidade de Massa & $2400 \mathrm{~kg} / \mathrm{m}^{3}$ \\
\hline \multirow{3}{*}{$\begin{array}{c}\text { Utotal }=\mathrm{R}_{\mathrm{SI}}+\mathrm{R}_{\text {Parede Interna }}+\mathrm{R}_{\text {Parede Leve }}+\mathrm{R}_{\mathrm{SE}} \\
\mathrm{U}(\mathrm{PL}+\mathrm{PI})=1,92 \mathrm{~W} / \mathrm{m} 2 \mathrm{~K} \\
(\text { Transmitância da parede de Alvenaria })\end{array}$} & \multirow{3}{*}{ Aglomerado } & Condutividade Térmica & $0,058 \mathrm{~W} / \mathrm{mK}$ \\
\hline & & Espessura & $0,0172 \mathrm{~m}$ \\
\hline & & Densidade & $338,46 \mathrm{~kg} / \mathrm{m}^{3}$ \\
\hline \multirow[t]{2}{*}{ Parede Pesada (PP) } & \multirow{3}{*}{ Concreto } & Condutividade Térmica & $1,75 \mathrm{~W} / \mathrm{mK}$ \\
\hline & & Espessura & $0,1 \mathrm{~m}$ \\
\hline \multirow{4}{*}{$\begin{array}{c}\text { Utotal }=\mathrm{R}_{\mathrm{SI}}+\mathrm{R}_{\text {Parede Interna }}+\mathrm{R}_{\text {Parede Leve }}+\mathrm{R}_{\mathrm{SE}} \\
\mathrm{U}(\mathrm{PP}+\mathrm{PI})=3,71 \mathrm{~W} / \mathrm{m} 2 \mathrm{~K} \\
\text { (Transmitância da Estrutura de Concreto } \\
\text { Armado })\end{array}$} & & Densidade de Massa & $2400 \mathrm{~kg} / \mathrm{m}^{3}$ \\
\hline & \multirow{3}{*}{ Reboco } & Condutividade Térmica & $1,15 \mathrm{~W} / \mathrm{mK}$ \\
\hline & & Espessura & $0,02 \mathrm{~m}$ \\
\hline & & Densidade de Massa & $2000 \mathrm{~kg} / \mathrm{m}^{3}$ \\
\hline
\end{tabular}

\subsubsection{Revisão e definição da DCI (equipamentos e iluminação)}

Utilizou-se a densidade de carga de equipamentos sugerida por CARLO (2008) com $60 \mathrm{~W} / \mathrm{m}^{2}$. Foram também utilizados os parâmetros de densidade de carga de 
equipamentos sugeridos pela NBR 16401 (ABNT, 2008) para comparação considerando: tipo de carga leve com $6 \mathrm{~W} / \mathrm{m}^{2}$, média com $11 \mathrm{~W} / \mathrm{m}^{2}$ e alta com $21 \mathrm{~W} / \mathrm{m}^{2}$.

A carga de iluminação utilizada por CARLO (2008) é de $21 \mathrm{~W} / \mathrm{m}^{2}$, no entanto foram utilizados os valores recomendados pelo RTQ-C (INMETRO, 2012) para a função do edifício, no caso hotel, sendo $10,8 \mathrm{~W} / \mathrm{m}^{2}$ para nível $\mathrm{A} ; 12,4 \mathrm{~W} / \mathrm{m}^{2}$ nível $\mathrm{B} ; 14 \mathrm{~W} / \mathrm{m}^{2}$ nível C e $15,7 \mathrm{~W} / \mathrm{m}^{2}$ nível $\mathrm{D}$.

\title{
2.2.2 Revisão e definição da DCI (equipamentos e iluminação)
}

$\mathrm{Na}$ configuração do controle ambiental o setpoint de aquecimento foi configurado para $22^{\circ} \mathrm{C}$ e o setback de aquecimento para $21^{\circ} \mathrm{C}$; o setpoint de resfriamento ajustado para $24^{\circ}$ e o setback de resfriamento para $25,5^{\circ} \mathrm{C}$. Os setpoints foram definidos com base na NBR 16401 (ABNT, 2008).

\subsection{Otimização das simulações}

A otimização das simulações pretende reduzir o tempo de configuração manual do elevado número de simulações que necessitam ser realizadas. Desenvolveu-se um analisador para gerar um novo processo de criação de novos IDFs (Input Data File), arquivos do programa Energy Plus/Design Builder. Para percorrer os IDFs base e modificá-los, para que cada novo IDF tenha suas propriedades de iluminação e de equipamentos modificadas automaticamente, foi desenvolvida a otimização das simulações.

As seguintes fórmulas são usadas nesse processo:

$$
\begin{gathered}
\text { Design Lighting Level }=\frac{D P I(\text { original })}{D C I(\text { original })} \times D P I(\text { novo }) \\
\text { Design Equipament Level }=\frac{D P I(\text { original })}{D C I(\text { original })} \times D P I(\text { novo })
\end{gathered}
$$

Também são inseridas ao final de cada novo IDF as seguintes rotinas de saída, para que o simulador gere resultados baseados na quantidade de energia gasta em iluminação, equipamentos, aquecimento e refrigeração.

\author{
Output: Meter, InteriorLight: Electricty, daily \\ Output: Meter, InteriorEquipament: Electricty, daily \\ Output: Meter, DistrictHeating: Facility, daily \\ Output: Meter, DistrictCooling: Facility, daily
}

Com todos os IDFs já gerados, a próxima etapa é a simulação. Nessa utiliza-se o software Building Controls Virtual Test Bed (BCVTB), para realizar co-simulações a partir de troca de mensagens em tempo real entre outros softwares. Na Figura 4 observa-se a relação entre o EnegyPlus e o Matlab.

Figura 4 - Interface BCVTB 


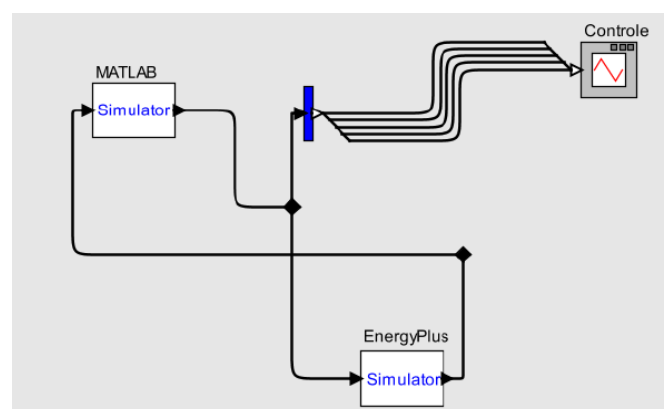

O EnergyPlus é um software desenvolvido para simulação de carga térmica e análise energética de edificações e seus sistemas. Para que a simulação seja realizada no $\mathrm{BCVTB}$, inicialmente configuramos o arquivo climático utilizado e as seguintes variáveis:

$$
\begin{aligned}
\text { startTime } & =0 \\
\text { finalTime } & =12 \times 31 \times 24 \times 3600 \\
\text { timeStep } & =3600
\end{aligned}
$$

Estas variáveis são respectivamente: tempo inicial, tempo final e tempo de uma simulação. Assim temos uma simulação ao longo de um ano, como resultados diários horários.

O BCVTB pode ser executado pela sua interface padrão ou também por linha de comando, e isto pode ser explorado por qualquer linguagem de programação.

Tanto BCVTB como o EnergyPlus trabalham com um IDF por vez. Para resolver essa limitação foi desenvolvida outra ferramenta para executar automaticamente o BCVTB para cada IDF e, após realizada a simulação, a ferramenta gerar tabelas e gráficos para uma melhor análise dos dados obtidos pela simulação.

\subsection{Simulação e comparação do consumo do caso base e do modelo de referência}

Com o modelo do caso base e o modelo de referência, com e sem pontes térmicas, modelados e configurados no DesignBuilder, deu-se inicio às simulações. Nos primeiros dados gerados foram utilizados os parâmetros de CARLO (2008). Após as primeiras simulações confirmou-se que a DCI de $60 \mathrm{~W} / \mathrm{m}^{2}$ estava fora dos parâmetros normativos. Passou-se a simular utilizando valores referenciados pela norma NBR 16401(ABNT, 2008) e ao RTQ-C (INMETRO, 2012) para os diferentes parâmetros de densidade de carga e de iluminação, gerando 36 configurações a serem simuladas, com e sem pontes térmicas.

\section{ANÁLISE DE RESULTADOS}

Os modelos foram simulados com e sem as pontes térmicas, variando as configurações de densidade de carga interna (DCI), a densidade de potência de iluminação (DPI) e o percentual de aberturas nas fachadas (PAFT) para as Zonas Bioclimáticas 1 a 6 . Os resultados das simulações foram organizados em tabelas (Tabela 4). A partir do estudo dessas tabelas gerou-se o Gráfico 1 que mostram as zonas bioclimáticas nas abscissas, numeradas de 1 a 6 , e a diferença entre o consumo anual dos modelos com e sem pontes térmicas em porcentagem nas ordenadas. Os resultados negativos se referem ao fato do modelo com pontes ter atingido um consumo superior ao modelo sem pontes. 
Gráfico 1 - Resultados das simulações para as seis zonas - consumo anual

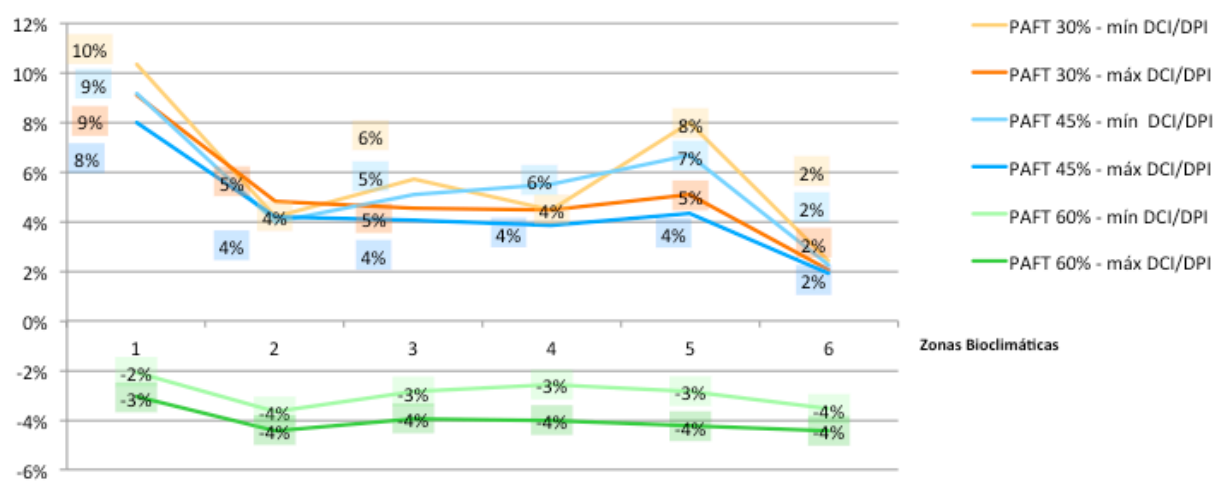

Quadro 3 - Resultado das Simulações para 4 Zonas - Consumo Anual

\begin{tabular}{|c|c|c|c|c|c|c|}
\hline $\begin{array}{c}\text { Zona } \\
\text { Bioclimática }\end{array}$ & $\begin{array}{c}\text { PAFT 30\% } \\
\text { mín } \\
\text { DCI/DPI }\end{array}$ & $\begin{array}{c}\text { PAFT 30\% } \\
\text { máx } \\
\text { DCI/DPI }\end{array}$ & $\begin{array}{c}\text { PAFT } \\
\text { 45\% mín } \\
\text { DCI/DPI }\end{array}$ & $\begin{array}{c}\text { PAFT 45\% } \\
\text { máx } \\
\text { DCI/DPI }\end{array}$ & $\begin{array}{c}\text { PAFT 60\% } \\
\text { mín } \\
\text { DCI/DPI }\end{array}$ & $\begin{array}{c}\text { PAFT 60\% } \\
\text { máx } \\
\text { DCI/DPI }\end{array}$ \\
\hline 1 & $10,33 \%$ & $9,13 \%$ & $9,15 \%$ & $7,96 \%$ & $-2,06 \%$ & $-3,09 \%$ \\
\hline 2 & $4,23 \%$ & $4,79 \%$ & $3,98 \%$ & $4,21 \%$ & $-3,66 \%$ & $-4,40 \%$ \\
\hline 3 & $5,73 \%$ & $4,57 \%$ & $5,06 \%$ & $4,06 \%$ & $-2,86 \%$ & $-3,93 \%$ \\
\hline 4 & $4,49 \%$ & $4,49 \%$ & $5,51 \%$ & $3,88 \%$ & $-2,56 \%$ & $-4,00 \%$ \\
\hline 5 & $7,97 \%$ & $5,06 \%$ & $6,66 \%$ & $4,36 \%$ & $-2,84 \%$ & $-4,25 \%$ \\
\hline 6 & $2,43 \%$ & $2,06 \%$ & $2,24 \%$ & $1,90 \%$ & $-3,56 \%$ & $-4,44 \%$ \\
\hline
\end{tabular}

O consumo total é a soma do consumo para aquecimento e refrigeração no período de um ano, ressalta-se que o consumo para resfriamento equivale a mais de $95 \%$ do consumo total de condicionamento artificial, tornando o consumo para aquecimento pouco significante. Entretanto, para aquecimento o modelo com as pontes térmicas tem um consumo em média $40 \%$ maior em todas as zonas.

Os resultados mostram que para os menores valores de DCI e DPI conjugados com maiores ganhos por condução pelos vidros, as pontes térmicas geram um aumento do consumo entre $2 \%$ e $3 \%$. E no caso das DCI e DPI altos, conjugados com maiores ganhos por condução pelos vidros, fazem com que o consumo aumente próximo de $4 \%$.

Nas zonas bioclimáticas 1 a 4 observa-se que para PAFT $30 \%$ e $45 \%$ os fechamentos com pontes térmicas proporcionam uma diminuição de até $10,33 \%$ no consumo total. Em relação ao PAFT $60 \%$ houve um aumento em todas as zonas de 3,09\% ; 4,40\% ; $3,93 \% ; 4,00 \%$ e $4,25 \%$ respectivamente. A zona bioclimática 1 teve a maior redução do consumo anual.

\section{CONCLUSÃO}

Inicialmente ressalta-se que a configuração proposta para a simulação das pontes térmicas utilizando o software DesignBuilder fazendo uso de sub-superfícies possibilitou a configuração do fechamento vertical com diferentes transmitâncias 
térmicas nas alvenarias e na estrutura do edifício, permitindo, portanto, a análise da variação da resistência térmica ao longo do plano vertical. Observando os resultados obtidos entende-se a importância da consideração das pontes térmicas no cálculo do desempenho energético de edifícios. Observou-se que a desconsideração das pontes para edificações de hotelaria com PAFT 30\% e 45\% em simulação energética implica em uma diminuição da estimativa de consumo, que dependendo da zona bioclimática pode chegar em $10 \%$. No caso de edificações com $60 \%$ de PAFT a não consideração da ponte térmica pode representar um aumento de até $4 \%$ no consumo dependendo da zona bioclimática.

\section{AGRADECIMENTOS}

Os autores do trabalho agradecem ao CNPq pelo apoio na realização da pesquisa.

\section{REFERÊNCIAS}

- NBR 15.220: Norma Brasileira de Desempenho Térmico de Edificações, Parte 3: Zoneamento Bioclimático Brasileiro e Diretrizes Construtivas para Habitações Unifamiliares de Interesse Social. Rio de Janeiro: ABNT, 2005.

. NBR 15.575: Desempenho de Edifícios habitacionais. Rio de Janeiro: ABNT, 2013.

ASSOCIAÇÃO BRASILEIRA DE NORMAS TÉCNICAS (ABNT). NBR-16401-1: Instalações de ar-condicionado -Sistemas centrais e unitário Parte 1: Projetos das instalações. Rio de Janeiro, 2008.

CARLO, Joyce Correna. Desenvolvimento de metodologia de avaliação da eficiência energética do envoltório de edificações não-residenciais. Tese de Doutorado. Universidade Federal de Santa Catarina. UFSC, 2008.

EVANS, John M.; de Schiller, Silvia.Verificación de puentes térmicos normas para definir soluciones admisibles. 2010. Avances en Energías Renovables y Medio Ambiente, v.14 p. 5158.

HAUPT, Wolfram. Skript Feuchsteschutz. Kassel Universität, Kassel, 2007.

INSTITUTO NACIONAL DE METROLOGIA, NORMALIZAÇÃO E QUALIDADE INDUSTRIAL (INMETRO). Requisitos Técnicos da Qualidade para o Nível de Eficiência Energética de Edifícios Comerciais, de Serviços e Públicos - RTQ-C. Eletrobrás, 2012.

INSTITUTO ARGENTINO DE NORMALIZACIÓN (IRAM 11549). Aislamiento térmico de edifícios. Argentina: IRAM. 2002.

MATOS, Michele. Simulação computacional do desempenho térmico de residências em Florianópolis utilizando a ventilação natural. Florianopolis, UFSC, 2007.

PEREIRA, Cláudia. A influência do envelope no desempenho térmico de edificações residenciais unifamiliares ocupadas e ventiladas naturalmente. 2009.124 f. Dissertação (Mestrado) - Programa de Pós-Graduação em Engenharia Civil, Universidade Federal de Santa Catarina, Florianópolis, 2009.

VALÉRIO, Jorge Gustavo Marques Alface Pereira. Avaliação do Impacte das Pontes Térmicas no Desempenho Térmico e Energético de Edifícios Residenciais Correntes. Instituto Técnico de Lisboa. 2007.

VENÂNCIO, Raoni. Investigando o projeto de envoltória: questionário sobre prática arquitetônica e desempenho térmico. XI Encontro Nacional de conforto no ambiente construído, Búzios RJ, 2011. 